\title{
Picturing unilateral spatial neglect: viewer versus object centred reference frames
}

\author{
Anjan Chatterjee
}

\begin{abstract}
Vision theorists postulate that knowledge of objects in space is shaped by different spatial coordinate systems. An object may be represented by its location in relation to the viewer, or it may be represented by its own intrinsic spatial properties. When patients with left sided neglect fail to respond to stimuli on the left, it is not clear whether "left" refers to a viewer or an object centred reference frame. To uncouple these two reference frames, eight patients with neglect were asked to centre lines and objects in photographs. Viewer centred neglect would result in images appearing on the right side of photographs and object centred neglect would result in images appearing on the left. Four patients demonstrated viewer centred neglect and three demonstrated object centred neglect. One patient had variable performance, perhaps resulting from competing effects of both viewer and object centred neglect. Stimuli characteristics did not affect the spatial coordinate system in which neglect occurred. These results suggest that viewer centred and object centred reference frames are functionally dissociable, and that patients may have spatial neglect predominantly in either coordinate system.
\end{abstract}

$(\mathcal{F}$ Neurol Neurosurg Psychiatry 1994;57:1236-1240)

Patients with left sided neglect fail to orient toward, respond to, or act on stimuli in the left side of space. ${ }^{1}$ When asked to bisect horizontal lines, these patients often place their mark to the right of the objective midpoint of the line, demonstrating neglect of the left side. Embedded within this simple, time honoured task is a potential confounding factor. The task does not discriminate between neglect of the left side of space into which the line extends, and neglect of the left side of the line itself. The magnitude of line bisection errors are influenced by line length as well as the spatial location of identical lines ${ }^{23}$ suggesting that neglect of the left side of lines and space frequently co-occur and may interact in complex ways. The purpose of this investigation was to try to determine if one form of neglect predominates.

Vision theorists postulate that the brain uses different spatial reference frames to code entities in space. ${ }^{46} \mathrm{~A}$ viewer centred reference frame locates an object from the vantage point of the viewer-that is, the left or right of, above or below the viewer. This reference frame may be further organised retinotopically, or with respect to the position of the viewer's head or trunk. An environment centred reference frame locates an object by its spatial environment. Thus a specific object in a room may be located with respect to its unique coordinates in the room and its spatial relation to other objects. This environment centred reference frame is unaffected by changes in the viewer's location. ${ }^{6}$ An object centred reference frame codes spatial aspects of an object itself, such as the object's intrinsic left and right, and top and bottom. This reference frame remains stable even when the object is moved about. These spatial reference frames normally operate in concert, permitting us to manoeuvre and interact with objects in our spatial environment.

Unilateral spatial neglect is commonly conceived of as a disorder of spatially directed attention..$^{7-10}$ With damage to the right hemisphere, the left hemisphere's vector of spatial attention, which is directed into right space, manifests itself as "neglect" of the left side. ${ }^{1}$ It is not clear, however, which left side (viewer, object, or environment) is actually being neglected. Environment centred reference frames, which potentially influence neglect, ${ }^{611-13}$ will not be discussed as the investigation reported here focuses on disentangling viewer from object centred coordinates.

This study considers several questions about unilateral spatial neglect and spatial reference frames. Do patients predominantly have viewer or object centred neglect? Do both viewer and object centred neglect occur? If there is a double dissociation between viewer and object centred neglect, what is the form of this dissociation? Do some patients demonstrate viewer and others object centred neglect? Or does the nature of the stimuli encountered determine the spatial coordinate system in which neglect occurs, so that the same patient demonstrates viewer centred neglect in one situation and object centred neglect in another? To explore these issues patients with left sided neglect performed a task designed to uncouple viewer and object centred reference frames. This uncoupling was achieved by instructing them to photograph objects so that these objects would appear exactly in the centre of the frame of the picture. If a patient were to neglect the left part of space in the view finder, then the objects would appear on the right side of the photograph. If they were to neglect the left side of the object, then the objects would appear on the left side of the picture (fig 1). Thus neglect in these two spatial coordinate systems predicts opposite patterns of performance. 
Figure 1 Prototypic effects of viewer centred (left) and object centred (right) neglect on placement of lines.
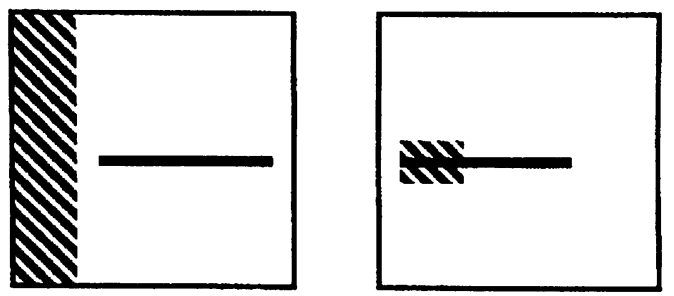

Subjects and methods

Eight patients from the Spain Rehabilitation Hospital with right hemisphere strokes were tested. Seven patients had had ischaemic strokes and one (patient 8) a haemorrhagic stroke. These patients showed evidence of left sided neglect as defined by screening measures described later. All the patients were right handed. There were five men and three women in this group. Their average age was 66 (range 53 to 76) years. All subjects had CT or MRI verification of their lesions. Their lesions were mapped in a standard manner ${ }^{14}$ and then graphically reconstructed to show the extent of the lesion along the cortical convexities. Patients were tested in my laboratory after consenting to participate in the study. They were seated in wheelchairs and all stimuli were centred in front of them on their midsagittal plane.

\section{SCREENING MEASURES}

Patients bisected three lines, $1 \mathrm{~mm}$ wide and 16,18 , or $20 \mathrm{~cm}$ long. Neglect on line bisection was defined as errors that were greater than an average of $0.5 \mathrm{~cm}$ to the right of the objective midpoint. Patients also performed two cancellation tasks. In one there were 32 "O"s distributed so that eight targets were randomly located within each quadrant. There were no distractors in this task. In the second, there were 24 target " $A$ "s distributed within 48 distractor letters, such that there were six targets and 12 distractors within each quadrant. Neglect in cancellations was defined as the omission of more targets on the left sides of both arrays than on the right. For drawing tasks, patients copied a simple line drawing of a house next to a fence and tree, and also drew a picture of a flower from memory. Neglect on these tasks was defined as not including left sided elements in either drawing. Patients were included in the study if they demonstrated neglect in at least one kind of task-line bisection, cancellation, or drawing. Visual fields to confrontation and extent of hemipareses were assessed by me. Table 1 shows performances of all eight patients.

\section{EXPERIMENT 1: LINE BISECTION}

All patients bisected lines $2 \mathrm{~cm}$ wide and 22, 23,24 , or $25 \mathrm{~cm}$ long twice for a total of eight bisections. Lines were horizontally presented in a random order and placed $30 \mathrm{~cm}$ in front of the patient centred on the mid-sagittal plane. Errors were recorded to the nearest $\mathrm{mm}$ deviation from the objective midpoint of the line.

\section{EXPERIMENT 2: PHOTOGRAPHING LINES}

This experiment was designed to uncouple viewer from object centred reference frames in
Table 1 Results of screening measures

\begin{tabular}{llllll}
\hline Patient & $L B$ & $C$ & $D$ & $V F D$ & $H P$ \\
1 & 23 & $07-05$ & + & + & + \\
2 & 09 & $12-08$ & + & + & + \\
3 & 09 & $32-23$ & + & + & + \\
4 & 02 & $28-17$ & - & - & + \\
5 & 43 & $11-06$ & + & - & + \\
6 & 25 & $25-16$ & + & + & \pm \\
7 & 45 & $04-02$ & + & + & + \\
8 & 56 & $08-08$ & + & - & + \\
\hline
\end{tabular}

$\mathrm{LB}=$ Line bisection, errors reported in average $\mathrm{mm}$ deviation to the right; $C=$ cancellation tasks, number of targets for two tasks reported (maximum 32-24); $\mathrm{D}=$ drawing task; VFD = visual field defect; $\mathrm{HP}=$ hemiparesis; + denotes presence of a deficit; - denotes absence of a deficit.

attending to a line. Black lines of the same measurements as in experiment 1 were fixed on a wall against a white background in a horizontal orientation. The lines were placed at eye level about $45 \mathrm{~cm}$ in front of the patients. In their right hand they held a Polaroid 600 SE Impulse auto focus camera. The view finder in this camera has no internal markings, such as a central focus ring. Each patient's right elbow was supported by a board attached to the armrests of their wheelchair. Their forearm was held in a near vertical position such that angular rotation of the forearm, pronation, or supination moved the image to the left or right of the view finder. Movement at the wrist was not restricted. Seven patients used their right eye to view the line through the camera view finder. One patient (patient 2) insisted on using his left eye, claiming that this was his "hunting eye". Eye movements were also not restricted. When the patient indicated that the line was accurately centred in the view finder, the investigator standing behind the subject pressed the shutter release.

Each line was randomly presented twice for a total of eight photographs. Deviations from the objective midpoint in the photographs were determined by subtracting the distance (in $\mathrm{mm}$ ) between the right border of the photograph and the right end of the line from the distance between the left end of the border to the left end of the line. A positive number indicates that the line was placed towards the right of the frame of the photograph, representing viewer centred neglect, and a negative number indicates that the line was placed to the left of the frame of the photograph, representing object centred neglect (fig 1).

EXPERIMENT 3: PHOTOGRAPHING OBJECTS

This experiment was conducted to determine if the nature of stimuli influences the reference frame in which neglect occurs. Confronting real objects might predispose attention to be deployed in an object centred reference frame. Six objects-a calculator, stapler, pen, scissors, remote control, and tape dispenserwere used as stimuli. These were placed on a table in front of the patients such that their long axes were horizontally oriented, and were centred on the patients' mid-sagittal plane. The objects were placed about $30 \mathrm{~cm}$ below patients' eye level and about $45 \mathrm{~cm}$ in front of the patients. The patients were instructed to first name the object before them and then photograph it as described in experiment 2 . 
Similar measurements of the location of the object within the photograph were made. One patient, (patient 7) refused further testing after experiment 2 .

Table 2 Deviation ( $\mathrm{mm}$ ) for each experiment

\begin{tabular}{llllll}
\hline Patient & Exp 1 & Exp 2 & Exp 3 & $R-L$ & $p$ Value \\
1 & 53 & +51 & +34 & $14-0$ & $<0.002$ \\
2 & 19 & +03 & +19 & $12-2$ & 0.038 \\
3 & 39 & +34 & +14 & $14-0$ & $<0.002$ \\
4 & 03 & +54 & +67 & $14-0$ & $<0.002$ \\
5 & 63 & -34 & -27 & $0-13^{\star}$ & $<0.002$ \\
6 & 44 & -08 & -02 & $2-12$ & 0.038 \\
7 & 35 & -15 & - & $0-8$ & 0.008 \\
8 & 95 & +15 & +06 & $8-5$ & NS \\
\hline
\end{tabular}

‡ The object in one photograph was accurately centred in the frame.

For experiments 2 and 3, + deviation means that lines or objects were photographed in the right of the frame, and deviation means that lines or objects were photographed in the left of the frame. The R-L column indicates the frequency with which lines or objects were placed to the right or left of the frame.

A
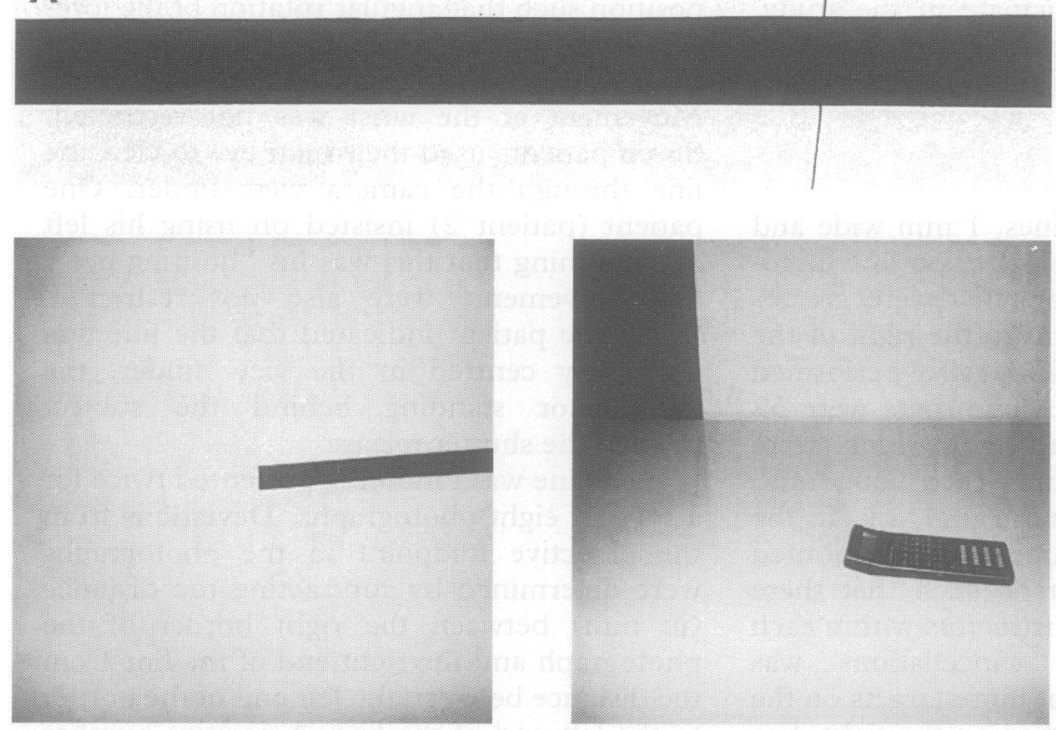

$\mathbf{B}$
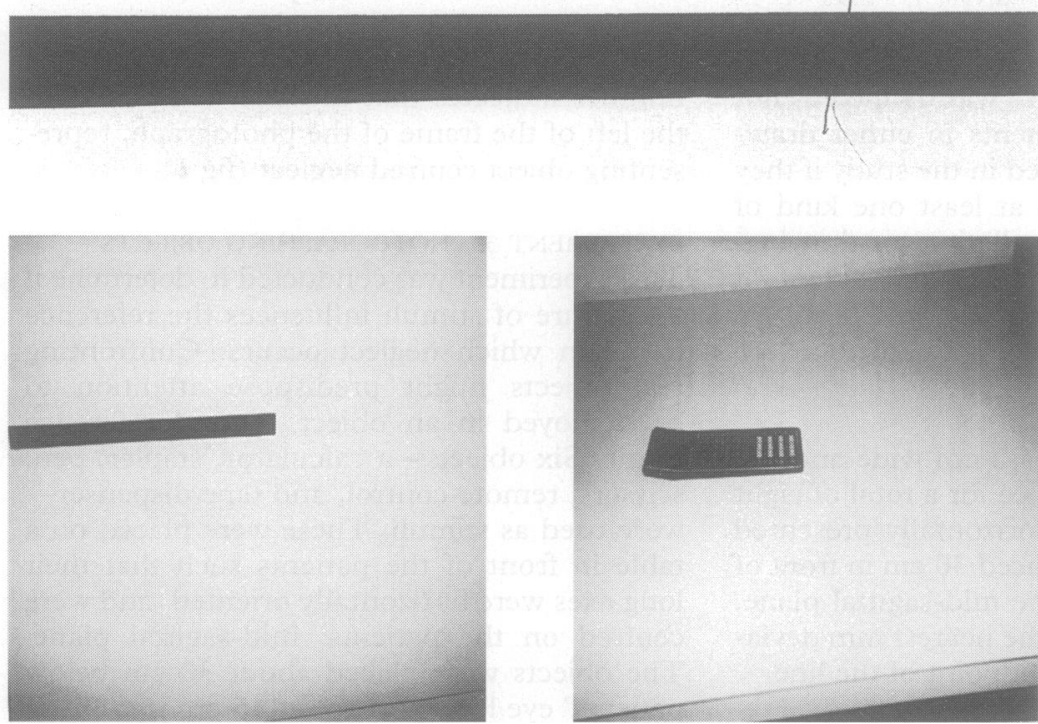

Figure 2 Examples of $(A)$ viewer centred neglect and $(B)$ object centred neglect. Line bisection performance (experiment 1) is shown at the top, with photographs of lines (experiment 2) and objects (calculator from experiment 3) shown below.

\section{Results}

Table 2 gives a summary of the results obtained. In Experiment 1, patient 4 (who had a frontal lesion) deviated to the right of the objective midpoint on line bisections by only $3 \mathrm{~mm}$. She did not show neglect on bisections in the screening tests despite omitting targets on cancellation tasks, a pattern previously reported in patients with frontal lesions. ${ }^{15}$ The other patients' bisections deviated to the right of the objective midline ranging from $19 \mathrm{~mm}$ to $95 \mathrm{~mm}$. When photographing lines and objects, some patients placed them to the left and others placed them to the right. In no instance did a patient's direction of errors (either left or right) differ between experiments 2 and 3, suggesting that stimuli characteristics (lines $v$ objects) did not affect performance. Data from these experiments were combined to assess statistical significance for the direction of errors by a two tailed binomial test. Four patients placed objects towards the right side of the photographs, showing viewer centred neglect (fig $2 \mathrm{~A}$ ). Three patients placed objects on the left side of the photographs, demonstrating object centred neglect (fig 2B). One patient (patient 8 ) had variable performance, sometimes placing objects to the left and sometimes to the right. The presence of a visual field defect was not associated with a specific direction of error in this group of patients. There were no apparent correlations between lesion location and the reference frame in which neglect was seen (fig 3).

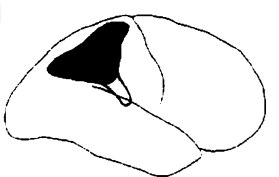

2
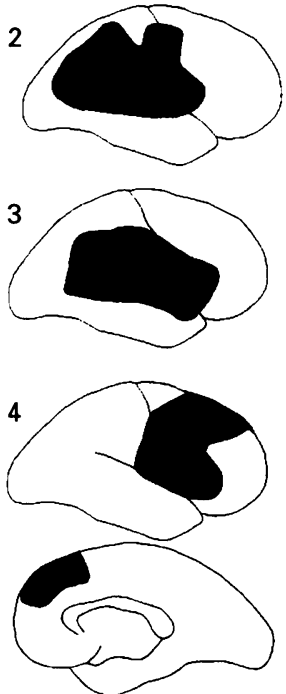

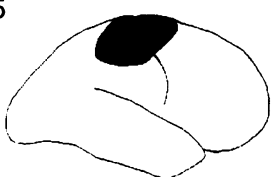

6

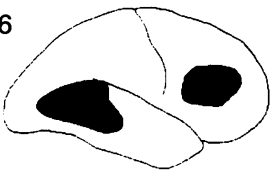

7
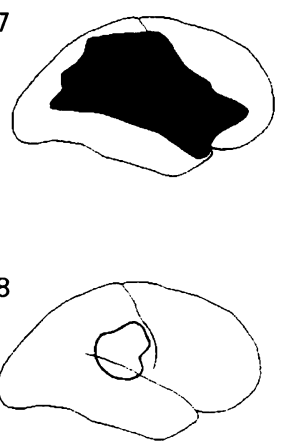

Figure 3 Graphic reconstructions of the patients' lesions. Cortical involvement is shown in black, and subcortical involvement without overlying cortical damage is shown in outline. Patient 1 had had a haemorrhagic stroke; all others had had ischaemic strokes. Patients 1, 2, 3, and 4 had viewer centred neglect, and patients 5, 6, and 7 had object centred neglect. Patient 1 had inconsistent performance. 


\section{Discussion}

This study confirms the existence of different spatial reference frames that vision theorists postulate by showing that neglect occurs in both viewer and object centred reference frames. These results extend findings of previous studies that have examined the relation between these two reference frames and neglect. Farah et $a l^{6}$ showed 10 patients with neglect for various line drawings (such as that of a rabbit) containing letters. The patients were asked to name the object and then read all the letters. By rotating the drawings, viewer and object centred reference frames were dissociated. The object centred reference frame did not have a discernable influence on which letters were neglected, whereas the viewer centred reference frame continued to influence the spatial distribution of neglect. Chatterjee et $a l^{3}$ examined the effects of hemispatial placement and attentional cueing on line bisection tasks. They found that the behaviour of a patient with neglect was affected by both conditions. When compared with normal subjects, however, the patient's performance was qualitatively different with changes in spatial location of the lines, and only quantitatively different when attention was cued to the left or right side of the lines themselves. These results were interpreted as showing a qualitative defect in a viewer centred reference frame and not an object centred reference frame. By contrast, Driver and Halligan $^{16}$ described a case in which neglect was determined by object centred coordinates. When the patient was required to match two objects, a decrement in performance was seen when the information required to make the discrimination fell on the object's left side. This decrement occurred even when the objects were rotated so that the critical area on the left side of the object was located to the right of the viewer. Caramazza and Hillis reported a case of a patient with a lesion in the left hemisphere who neglected the right side of words in reading. ${ }^{17}$ This patient continued to neglect terminal portions of words (object centred) even when these words were presented in mirror reversed form so that the neglected portion of the word fell on the left side of the viewer.

The study of Farah et $a l^{6}$ raises doubts about whether object centred neglect occurs at all. The other three studies report single cases, limiting their generalisability. The stimuli used in these studies were very different. It is possible that the individual results, whether implicating viewer or object centred neglect, were a consequence of the kinds of stimuli and testing procedures used. None of the studies showed a double dissociation of viewer and object centred neglect by the same testing procedures. The strongest evidence suggestive of the occurrence of either viewer or object centred neglect comes from drawings by patients with neglect. In copying tasks patients may either neglect objects on the left side of an array or the left side of objects in the array. ${ }^{18} 19$ The within patient consistency, however, of these drawing performances is need of further investigation.

This study supports the existence of a double dissociation between viewer and object centred neglect. All the patients had left sided neglect as traditionally defined. When required to place lines and objects in the centre of photographs their performances, however, diverged. Some patients placed objects on the left side of the photograph, demonstrating neglect of the left side of objects. Others placed objects on the right side of the photograph, demonstrating neglect of the left side of their view. These placements resulted primarily from patients' angular rotations of the forearm making directional hypokinesia an unlikely explanation for these findings. As lateral movements of the wrist were not specifically constrained, this possibility cannot, however, be completely eliminated.

The results of this study also distinguish between two alternative ways in which a functional dissociation between viewer and object centred reference frames could occur. Attentional activation of these reference frames could be driven by the nature of the sensory stimuli in a "bottom up" manner. The same patient might then demonstrate viewer centered neglect in situations that would normally activate viewer coordinates and object centred neglect in situations that would normally activate object coordinates. Alternatively, attentional mechanisms themselves might be organised along different reference frames, and could be selectively damaged in a "top down" manner. Then some patients would have viewer centred neglect and others object centred neglect independent of stimuli encountered. Experiments 2 and 3 were designed to distinguish between these alternatives. A line is arguably a poor object, as it only has the property of two dimensional linear extension, without the richness of critically defining features. The objects in experiment 3 were real, with their associated shapes, colours and functions, that patients named before taking their picture. If the spatial coordinate system in which attention is deployed is determined by the nature of the stimuli, one would predict that photographing lines might induce viewer centred neglect and photographing real objects might induce object centred neglect. In this study, the nature of the stimuli did not influence the spatial coordinate system in which neglect was found. Rather, patients demonstrated the same form of neglect whether confronting lines or objects. These findings suggest that attentional systems themselves are organised along spatial reference frames and that these distinct attentional systems may be disparately damaged. These results are in accord with Halligan and Marshall's view that the neglect syndrome encompasses quite different underlying impairment. ${ }^{20}$ Even patients with similar performances on standard line bisections further fractionate when asked to photograph these same stimuli (fig 2).

Two patients at the extremes of performance were interesting. Patient 4 did not have 
neglect on line bisections, deviating only an average of $3 \mathrm{~mm}$ to the right of the true midpoint. Yet she demonstrated substantial viewer centred neglect. She may have had a completely preserved object centred reference frame allowing her to bisect accurately when only confronting the object, but when attempting to place this object within a photographic frame her abnormal viewer centred frame became manifest. She was the only patient with a lesion confined to the dorsolateral and medial frontal lobe. At the other extreme, patient 8 made the largest errors on line bisections, deviating an average of $95 \mathrm{~mm}$ to the right of the true midpoint. She was the only subject who was inconsistent in where she placed lines and objects in her photographs. She may have had similar degrees of both viewer and object centred neglect. Thus when these different reference frames produced biases in opposite directions her performance collapsed into inconsistency, and when both reference frames produced biases in the same direction her performance deviated dramatically to the right. She was the only patient with a large putaminal haemorrhage extending into the adjacent white matter. The interpretations of these two patients' behaviour remains tentative, and require confirmation from future studies. Additional patients with frontal lobe and subcortical lesions will have to be studied to determine if these brain regions have unique contributions to spatial coordinate systems.

A final comment about the brain-behaviour relation in these patients needs to be made. It is a tenet of vision research that visual information is processed in a modular fashion. ${ }^{21} \mathrm{~A}$ dorsal "where" stream processes visual information to spatially locate objects. A ventral "what" stream processes visual information to identify objects. One might expect that the dorsal where stream is important in constructing a viewer centred reference frame and the ventral what stream is important in constructing object centred reference frames. If attention and higher level perceptual processing are functionally linked, then they might also have close anatomical corollaries. Then lesions predominantly affecting the dorsal stream would produce viewer centred neglect and lesions predominantly affecting the ventral stream would produce object centred neglect. The lesions of the patients in this study did not support this notion that the anatomical substrate for attentional mediation of these spatial coordinate systems directly corresponds to the dorsal and ventral anatomical divisions of perceptual processing. These patients had relatively large lesions, however. Further studies with patients with strategically placed circumscribed lesions may be needed to determine if damage to the attentional mediation of different reference frames results from damage to distinct cerebral loci.

In summary, this study confirms the notion that visual information is organised in the brain along viewer and object centred spatial reference frames. Brain damage that results in left sided neglect may disparately impair spatially directed attention in either coordinate system. Some patients neglect the left side of their view, others the left side of objects they encounter, and perhaps some neglect both.

I thank Lisa Santer, MD for helpful editorial comments and Thomas Groomes, MD for patient referrals. This work was supported by an NIH-NINDS Clinical Investigator supported by an NIH-NINDS

1 Heilman KM, Watson RT, Valenstein E. Neglect and related disorders. In: Heilman KM, Valenstein E, eds. relinical neuropsychology. 2nd ed. New York: Oxford University Press, 1985:243-93.

2 Marshall JC, Halligan PW. Line bisection in a case of visual neglect: psychophysical studies with implication for theory. Cognitive Neuropsychology 1990;7:107-30.

3 Chatterjee A, Mennemeier M, Heilman KM. The psychophysical power law and unilateral spatial neglect. Brain and Cognition 1994;25:92-107.

4 Marr D. Vision. A computational investigation into the human representation and processing of visual information. New York: WH Freeman and Co, 1982:397.

5 Feldman JA. Four frames suffice: a provisional model of vision and space. Behavioural and Brain Sciences 1985; 8:265-89.

6 Farah MJ, Brun JL, Wong AB, Wallace MA, Carpenter PA. Frames of reference for allocating attention to space: evidence from the neglect syndrome. Neuropsychologia 1990;28:335-47.

7 Brain WR. Visual disorientation with special reference to lesions of the right hemisphere. Brain 1941;64:224-72.

8 Kinsbourne $M$. A model for the mechanisms of unilateral neglect of space. Transactions of the American Neurological Association 1970;95:143-7.

9 Heilman KM, Valenstein E. Frontal lobe neglect in man. Neurology 1972;22:660-4.

10 Mesulam M-M. A cortical network for directed attention and unilateral neglect. Ann Neurol 1981;10:309-25.

11 Calvanio R, Petrone PN, Levine DN. Left visual spatial neglect is both environment-centered and body-centered. Neurology 1987;37:1179-83.

12 Ladavas E. Is the hemispatial damage produced by right parietal lobe damage associated with retinal or gravitational coordiantes. Brain 1987;110:167-80.

13 Mennemeier M, Chatterjee A, Heilman K. Neglect relative to gravity [abstract]. $\mathcal{F}$ Clin Exp Neuropsychol 1992; tive to
$14: 85$.

14 Damasio H, Damasio AR. Lesion analysis in neuropsychology. New York: Oxford University Press, 1989: psych.

15 Binder J, Marshall R, Lazar R, Benjamin J, Mohr J. Distinct syndromes of hemineglect. Arch Neurol 1992; 49:1187-94

16 Driver J, Halligan PW. Can visual neglect operate in object-centered coordinates? An affirmative single-case study. Cognitive Neuropsychology 1991;8:475-496.

17 Caramazza A, Hillis AE. Levels of representation, coordiante frames, and unilateral neglect. Cognitive Neuropsychology 1990;7:391-445.

18 Gainotti G, Messerli P, Tissot R. Qualitative analysis of unilateral spatial neglect in relation to laterality of cerebral lesions. I Neurol Neurosurg Psychiatry 1972; 35:545-50.

19 Marshall JC, Halligan PW. Visuo-spatial neglect: a new copying test to assess perceptual parsing. F Neurol 1993; 240:37-40.

20 Halligan PW, Marshall JC. Left visuo-spatial neglect: a meaningless entity? Cortex 1992;28:525-35.

21 Ungerleider LG, Mishkin M. Two cortical visual systems. In: Ingle DJ, Goodale MA, Mansfield RJW, eds. Analysis of visual behavior. Cambridge, MA: MIT Press, 1982: 549-86. 